\title{
Three-Component Synthesis of Some New Coumarin Derivatives as Anticancer Agents
}

\author{
Latifah A. Alshabanah ${ }^{1}$, Laila A. Al-Mutabagani ${ }^{1}$, Sobhi M. Gomha ${ }^{2,3 * t}$ and Hoda A. Ahmed ${ }^{2,4}$ \\ ${ }^{1}$ Department of Chemistry, College of Science, Princess Nourah Bint Abdulrahman University, Riyadh, Saudi Arabia, ${ }^{2}$ Department \\ of Chemistry, Faculty of Science, Cairo University, Cairo, Egypt, ${ }^{3}$ Chemistry Department, Faculty of Science, Islamic University of \\ Madinah, Madinah, Saudi Arabia, ${ }^{4}$ Chemistry Department, College of Sciences, Taibah University, Yanbu, Saudi Arabia
}

OPEN ACCESS

Edited by:

Angela Stefanachi,

Angela Stefanachi, Italy

Reviewed by:

Florenci Vicent González, University of Jaume I, Spain

Paras Nath Yadav,

Tribhuvan University, Nepal

*Correspondence:

Sobhi M. Gomha

s.m.gomha@gmail.com

smgomha@iu.edu.sa

tORCID:

Sobhi M. Gomha orcid.org/0000-0001-6744-7817

Specialty section:

This article was submitted to

Organic Chemistry,

a section of the journal

Frontiers in Chemistry

Received: 21 August 2021 Accepted: 13 December 2021 Published: 25 January 2022

Citation:

Alshabanah LA, Al-Mutabagani LA, Gomha SM and Ahmed HA (2022) Three-Component Synthesis of Some New Coumarin Derivatives as Anticancer Agents.

Front. Chem. 9:762248.

doi: 10.3389/fchem.2021.762248
A three-component reaction for the synthesis of novel 3-heteroaryl-coumarin utilizing acetylcoumarin synthon under ultrasonic irradiation was developed using chitosan-grafted poly(vinylpyridine) as an eco-friendly catalyst. The process is a simple, facile, efficient procedure for the preparation of compounds displaying a thiazole ring linked to coumarin moiety. Moreover, all the products were evaluated for their anticancer activities against HEPG2-1. The results revealed that three new compounds showed promising anticancer activities.

Keywords: acetylcoumarin, hydrazonoyl halides, multicomponent synthesis, ultrasonic radiation, anticancer

\section{INTRODUCTION}

Today, the second cause of death in the world is cancer (Gomes et al., 2011). Chemotherapy has become one of the important methods for cancer treatment. The identification of novel, more potent, selective, and less toxic antitumor agents is the main aim for the researchers due to its widespread, rapid development and the severe infection of the tumor diseases. In the efforts to offer suitable anticancer drugs, medicinal researchers have focused on coumarin systems.

Coumarin is a naturally occurring material as well as a versatile synthetic scaffold exhibiting a wide spectrum of biological impacts including potential anticancer activities (Vosooghi et al., 2010) such as seselin (skin cancer) (Nishino et al., 1990), acronycin (lung, colon, and ovarian cancers) (Thakur et al., 2015), calanone (leukemia and cervical carcinoma) (Emami and Dadashpour, 2015), and tephrosin (lung cancer) (Lin et al., 2014). In addition, coumarin derivatives have a tremendous ability to regulate a diverse range of cellular pathways that can be explored for their selective anticancer activities (Geisler et al., 2011; Saidu et al., 2012). Moreover, the biological evaluations of coumarins revealed that the engrossment of innumerable pathways via coumarins acts as anticancer agents. They target a number of pathways in cancer like as kinase inhibition, cell cycle arrest, heat shock protein (HSP90) inhibition, angiogenesis inhibition, monocarboxylate transporters inhibition, antimitotic activity, carbonic anhydrase inhibition, telomerase inhibition, aromatase inhibition, and sulfatase inhibition (Ekowati et al., 2010; Li et al., 2010; Wang et al., 2011; Bhattarai et al., 2021).

Many investigations indicated that 1,3-thiazole derivatives possessed potential anticancer activities against various cancer types (Figure 1) (Luzina and Popov, 2009; Kashyap et al., 2018; Popsavin et al., 2007; Mavrova et al., 2009). Moreover, researchers found that the 1,3,4-thiadiazole

Abbreviations: DMF, dimethylformamide; DMSO- $d_{6}$, deuterated dimethylsulfoxide; EtOH, ethanol; g-chitosan, chitosangrafted poly(vinylpyridine); HEPG2-1, liver carcinoma cell line; MCR, multicomponent reactions; MTT, mean transit time (brain tissue blood flow); TEA, triethylamine; TLC, thin-layer chromatography; USI, ultrasonic irradiation. 


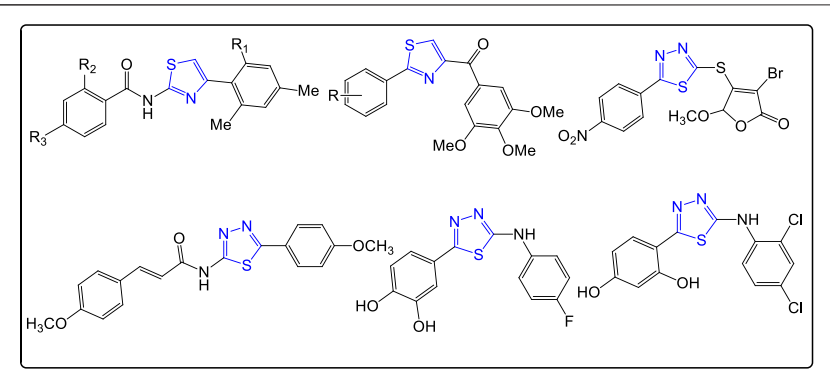

FIGURE 1 | Lead compounds among thiazoles and thiadiazoles with anticancer activities.

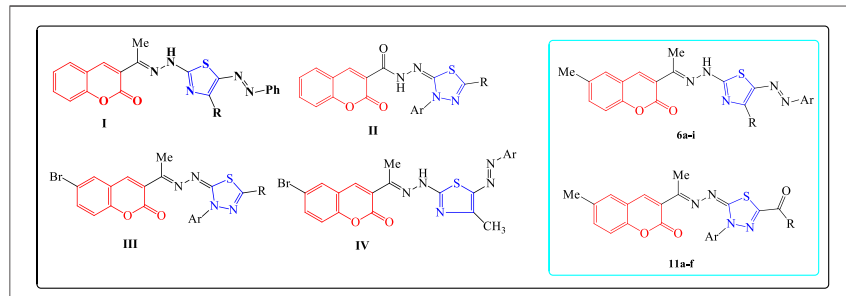

FIGURE 2 | Coumarin-based thiazoles or thiadiazoles with anticancer activities and the targeted compounds.

derivatives exhibited anticancer activities with excellent $\mathrm{IG}_{50}$ and IC $_{50}$ (Figure 1) (Liaras et al., 2018; Matysiak and Opolski, 2006; Kumar et al., 2010; Bhole and Bhusari, 2010). Many reports also revealed that the link between thiazole or thiadiazole groups and the coumarin moiety has broad spectra of pharmacological activities especially antitumor activities (Figure 2) (Gomha et al., 2015a; Gomha et al., 2015b; Gomha and Abdel-Aziz, 2015).

Multicomponent reactions (MCR) are one-pot mechanisms that produce a single product with at least three components, combining most or all of the starting materials (Marcaccini et al., 2003; Shaabani et al., 2008; Bachman et al., 2012; Gomha and Riyadh, 2014; Mamaghani and Hossein Nia, 2021), attributed to their reaction simplicity and high efficiency compared with multistage procedures. Furthermore, the ultrasound irradiation technique has been accomplished as an efficient heating source for organic reactions in synthetic organic chemistry. The main advantages of ultrasound-assisted reactions are shorter reaction time, simple experimental procedure, high yields, more selectivity, and clean processes (Xu et al., 2007; Jarag et al., 2011; Singh et al., 2013). One of the beneficial effects of ultrasound irradiation is playing a vital role in chemistry, especially in cases where classical tools require drastic conditions or long reactions times (Cravotto and Cintas, 2006; Cravotto et al., 2010; Pizzuti et al., 2010).

Chitosan is generated by alkaline hydrolysis of chitin (Gupta and Ravi Kumar, 2000). It is the naturally occurring copolymer polysaccharide including both glucosamine and acetylglucosamine units. Chitosan is used, in heterocyclic synthesis, as a heterogeneous phase transfer basic biocatalyst (Guibal, 2005; Qin et al., 2012; Watile and Bhanage, 2012; Alshabanah et al., 2021). Chitosan's key drawback is that it is extremely hygroscopic and can form gels, making it difficult to recycle from the reaction mixture. To overcome this limitation, chitosan-grafted poly(vinylpyridine) has been used as a basic biocatalyst with high catalytic activities (Fu et al., 2011), which can be easily recycled and has a better basic character owing to the presence of pyridine rings.

In the light of the above findings and in continuation of our efforts to synthesize new antitumor compounds (Abbas et al., 2015; Gomha et al., 2015c; Dawood and Gomha, 2015; Gomha et al., 2016a; Gomha et al., 2016b; Gomha et al., 2017a; Gomha et al., 2017b; Tao et al., 2018; Gomha et al., 2021), the aim of the present work is to design and synthesize thiazoles and thiadiazoles linked to position 3 of coumarin as novel 3-azolylcoumarins as expected anticancer agents, utilizing sonication technique and using chitosan-grafted poly(vinylpyridine) as an eco-friendly catalyst.

\section{METHODS AND METHODOLOGY}

The mass spectra were recorded on GCMS-Q1000-EX Shimadzu and GCMS 5988-A HP spectrometers, and the ionizing voltage was $70 \mathrm{eV}$ (Tokyo, Japan). The IR spectra were recorded in potassium bromide discs on Shimadzu FT IR 8101 PC infrared spectrophotometer (Shimadzu, Tokyo, Japan). The ${ }^{1} \mathrm{H}$ - and ${ }^{13} \mathrm{C}-\mathrm{NMR}$ spectra were recorded on Varian Mercury VXR-300 spectrometer $\left(300 \mathrm{MHz}\right.$ for ${ }^{1} \mathrm{H}-\mathrm{NMR}$ and $75 \mathrm{MHz}$ for $\left.{ }^{13} \mathrm{C}-\mathrm{NMR}\right)$, and the chemical shifts were related to those of the solvent DMSO- $d_{6}$ (Varian, Inc., Karlsruhe, Germany). All reactions were followed by thin-layer chromatography (TLC) (silica gel, Aluminum Sheets 60 F254, Merck, Cairo, Egypt). Elemental analyses were carried out at the Microanalytical Centre of Cairo University, Giza, Egypt. Sonication was performed in Shanghai Branson-CQX ultrasonic cleaner at a frequency of $40 \mathrm{kHz}$, and ultrasonic power was kept at $250 \mathrm{~W}$.

\section{General Procedure for Synthesis of 1,3-Thiazole Derivatives 6a-i}

Method A: Triethylamine (TEA) $(0.07 \mathrm{ml})$ was added to a mixture of the appropriate hydrazonoyl halides $5 \mathbf{a}-\mathbf{i}$ (1 mmol), thiosemicarbazide 4 (0.091 g, $1 \mathrm{mmol})$, and 3-acetyl-6-methyl$2 \mathrm{H}$-chromen-2-one (3) (0.202 g, $1 \mathrm{mmol})$ in $20 \mathrm{ml}$ of dioxane. The formed solution was irradiated by an ultrasonic generator in a water bath at $50^{\circ} \mathrm{C}$ for $20-60 \mathrm{~min}$. Irradiation was continued till all of the starting materials have disappeared and the product was formed, monitored by TLC. The red precipitate that formed after cooling was filtered off, washed with $\mathrm{EtOH}$, dried, and recrystallized from dimethylformamide (DMF) to give the corresponding thiazoles $\mathbf{6 a}-\mathbf{i}$. The physical constants of products $\mathbf{6 a - i}$ are listed below.

Method B: A mixture of equimolar amounts of 3, 4 and the appropriate $5 \mathbf{a}-\mathbf{i}(1 \mathrm{mmol}$ each) in dioxane $(10 \mathrm{ml})$ containing chitosan (10 mol\%) was irradiated by an ultrasonic generator in a water bath at $50^{\circ} \mathrm{C}$ for $20-60 \mathrm{~min}$ (monitored by TLC). The hot solution was filtered to remove chitosan, and excess solvent was removed under reduced pressure. The reaction mixture was triturated with methanol, and the product separated was 


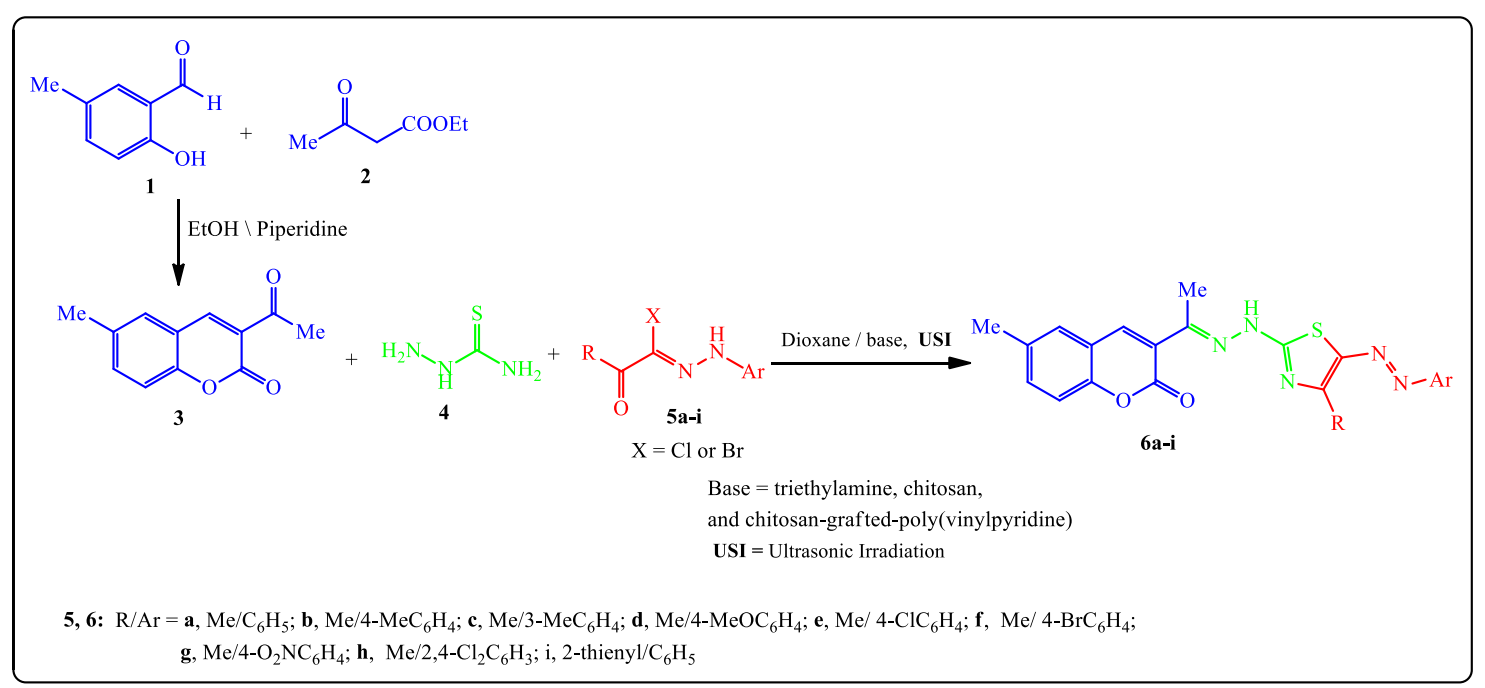

SCHEME 1 | Synthesis of arylazothiazole derivatives $\mathbf{6 a - i}$.

filtered, washed with methanol, dried, and recrystallized from DMF to give compounds $\mathbf{6 a - i}$.

Method C: The same procedure in method B using grafted chitosan (10 mol\%) instead of chitosan.

\section{6-Methyl-3-(1-(2-(4-methyl-5-(phenyldiazenyl) thiazol-2-yl)hydrazono)ethyl)-2H-chromen-2-one (6a)} Red solid, m.p. $183^{\circ} \mathrm{C}-185^{\circ} \mathrm{C}$; IR (KBr) $v \mathrm{~cm}^{-1}: 3,427(\mathrm{NH})$, $1,724(\mathrm{C}=\mathrm{O}), 1,602(\mathrm{C}=\mathrm{N}) ;{ }^{1} \mathrm{H}-\mathrm{NMR}\left(\mathrm{DMSO}-d_{6}\right) \delta: 2.13(\mathrm{~s}$, $\left.3 \mathrm{H}, \mathrm{CH}_{3}\right), 2.44\left(\mathrm{~s}, 3 \mathrm{H}, \mathrm{CH}_{3}\right), 2.67\left(\mathrm{~s}, 3 \mathrm{H}, \mathrm{CH}_{3}\right), 6.63-7.83(\mathrm{~m}$, $8 \mathrm{H}, \mathrm{Ar}-\mathrm{H}), 8.29$ (s, $1 \mathrm{H}$, coumarin-H4), $11.16(\mathrm{~s}, \mathrm{br}, 1 \mathrm{H}, \mathrm{NH})$ ppm; ${ }^{13} \mathrm{C}$-NMR (DMSO- $\left.d_{6}\right): \delta 11.3,16.2,20.1\left(\mathrm{CH}_{3}\right), 115.6$, $118.5,125.4,127.5,127.8,128.2,128.7,132.9,133.1,133.5$, $133.9,140.5,140.8,146.0,151.4(\mathrm{Ar}-\mathrm{C}), 168.1(\mathrm{C}=\mathrm{O}) \mathrm{ppm}$; MS $m / z(\%): 417.13\left(\mathrm{M}^{+}, 63\right), 351$ (42), 299 (69), 247 (41), 93 (22), 80 (100), 64 (70). Anal. calcd for $\mathrm{C}_{22} \mathrm{H}_{19} \mathrm{~N}_{5} \mathrm{O}_{2} \mathrm{~S}$ (417.49): C, 63.29; H, 4.59; N, 16.78. Found: C, 63.21; H, 4.50; N, $16.69 \%$.

\section{6-Methyl-3-(1-(2-(4-methyl-5-(p-tolyldiazenyl)} thiazol-2-yl)hydrazono)ethyl)-2H-chromen-2-one (6b) Red solid, m.p. $170^{\circ} \mathrm{C}-172^{\circ} \mathrm{C}$; IR (KBr) $v \mathrm{~cm}^{-1}: 3,428(\mathrm{NH}), 1,729$ $(\mathrm{C}=\mathrm{O}), 1,600(\mathrm{C}=\mathrm{N}) ;{ }^{1} \mathrm{H}-\mathrm{NMR}\left(\mathrm{DMSO}-d_{6}\right) \delta: 2.13\left(\mathrm{~s}, 3 \mathrm{H}, \mathrm{CH}_{3}\right)$, $2.38\left(\mathrm{~s}, 3 \mathrm{H}, \mathrm{CH}_{3}\right), 2.44\left(\mathrm{~s}, 3 \mathrm{H}, \mathrm{CH}_{3}\right), 2.73\left(\mathrm{~s}, 3 \mathrm{H}, \mathrm{CH}_{3}\right), 6.65-7.71$ (m, 7H, Ar-H), 8.29 (s, 1H, coumarin-H4), 11.18 (s, br, 1H, NH) ppm; MS m/z (\%): 431 ( $\left.\mathrm{M}^{+}, 100\right), 365$ (40), 313 (26), 214 (27), 106 (53), 90 (100), 65 (70). Anal. calcd for $\mathrm{C}_{23} \mathrm{H}_{21} \mathrm{~N}_{5} \mathrm{O}_{2} \mathrm{~S}$ (431.51): C, 64.02; H, 4.91; N, 16.23. Found: C, 64.00; H, 4.83; N, $16.14 \%$.

\section{6-Methyl-3-(1-(2-(4-methyl-5-( $m$-tolyldiazenyl)} thiazol-2-yl)hydrazono)ethyl)-2H-chromen-2-one (6c) Red solid, m.p. $183^{\circ} \mathrm{C}-185^{\circ} \mathrm{C}$; IR (KBr) $v \mathrm{~cm}^{-1}: 3,426(\mathrm{NH}), 1,723$ $(\mathrm{C}=\mathrm{O}), 1,609(\mathrm{C}=\mathrm{N}) ;{ }^{1} \mathrm{H}-\mathrm{NMR}\left(\mathrm{DMSO}-d_{6}\right) \delta: 2.13\left(\mathrm{~s}, 3 \mathrm{H}, \mathrm{CH}_{3}\right)$, $2.26\left(\mathrm{~s}, 3 \mathrm{H}, \mathrm{CH}_{3}\right), 2.39$ (s, 3H, $\left.\mathrm{CH}_{3}\right), 2.75\left(\mathrm{~s}, 3 \mathrm{H}, \mathrm{CH}_{3}\right), 6.65-7.66$ $(\mathrm{m}, 7 \mathrm{H}, \mathrm{Ar}-\mathrm{H}), 8.29$ (s, 1H, coumarin-H4), $10.86(\mathrm{~s}, \mathrm{br}, 1 \mathrm{H}, \mathrm{NH})$ ppm; MS m/z (\%): 431 (83), 384 (41), 214 (23), 106 (87), 90 (100), 65 (76). Anal. calcd for $\mathrm{C}_{23} \mathrm{H}_{21} \mathrm{~N}_{5} \mathrm{O}_{2} \mathrm{~S}$ (431.51): C, 64.02; $\mathrm{H}, 4.91$; N, 16.23. Found: C, 64.01; H, 4.84; N, 16.06\%.

TABLE 1 | Comparison of synthesis of thiazolylcoumarines $\mathbf{6 a - i}$ under ultrasound irradiation using different basic catalysts on the time of reaction and the yield\% of the products.

\begin{tabular}{|c|c|c|c|c|c|c|}
\hline \multirow[t]{2}{*}{ Compound no } & \multicolumn{2}{|c|}{ TEA } & \multicolumn{2}{|c|}{ Chitosan } & \multicolumn{2}{|c|}{ g-Chitosan ${ }^{a}$} \\
\hline & Time (min) & (\%) Yield & Time (min) & (\%) Yield & Time (min) & (\%) Yield \\
\hline $6 a$ & 50 & 72 & 41 & 79 & 20 & 93 \\
\hline $6 c$ & 56 & 73 & 46 & 84 & 25 & 91 \\
\hline $6 d$ & 53 & 72 & 43 & 84 & 28 & 94 \\
\hline $6 e$ & 52 & 72 & 36 & 86 & 19 & 89 \\
\hline $6 h$ & 50 & 72 & 37 & 84 & 26 & 90 \\
\hline $6 i$ & 45 & 72 & 37 & 86 & 28 & 92 \\
\hline
\end{tabular}

${ }^{a}$ g-chitosan, chitosan-grafted poly(vinylpyridine); TEA, triethylamine. 


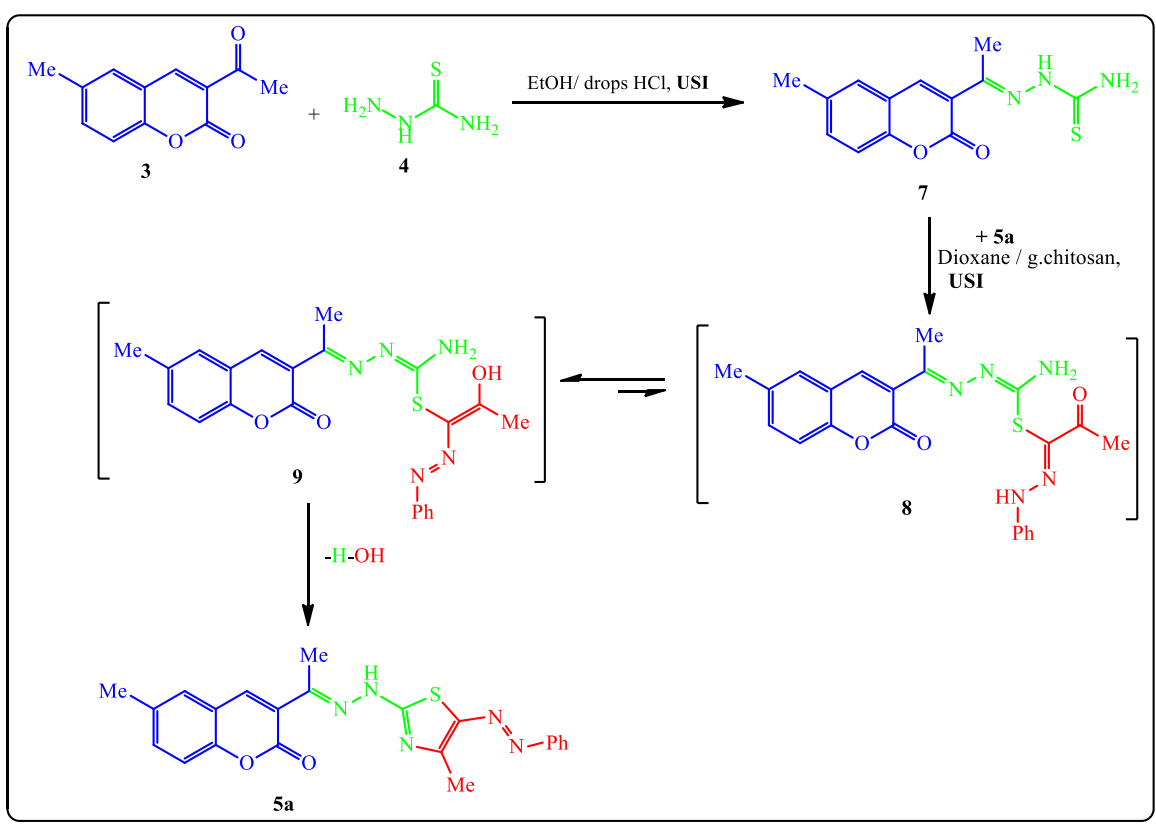

SCHEME 2 | Alternative synthesis of phenyazothiazole derivative $\mathbf{5 a}$

3-(1-(2-(5-((4-Methoxyphenyl) diazenyl)-4-methylthiazol-2-yl)hydrazono) ethyl)-6-methyl-2H-chromen-2-one (6d)

Red solid, m.p. $162^{\circ} \mathrm{C}-164^{\circ} \mathrm{C}$; IR ( $\left.\mathrm{KBr}\right) \nu \mathrm{cm}^{-1}: 3,416(\mathrm{NH}), 1,724$ $(\mathrm{C}=\mathrm{O}), 1,604(\mathrm{C}=\mathrm{N}){ }^{1} \mathrm{H}-\mathrm{NMR}\left(\mathrm{DMSO}-d_{6}\right) \delta: 2.13\left(\mathrm{~s}, 3 \mathrm{H}, \mathrm{CH}_{3}\right)$, $2.38\left(\mathrm{~s}, 3 \mathrm{H}, \mathrm{CH}_{3}\right), 2.70\left(\mathrm{~s}, 3 \mathrm{H}, \mathrm{CH}_{3}\right), 3.84\left(\mathrm{~s}, 3 \mathrm{H}, \mathrm{OCH}_{3}\right)$, 6.64-7.77 (m, 7H, Ar-H), 8.29 (s, 1H, coumarin-H4), 11.15 (s, br, $1 \mathrm{H}, \mathrm{NH}) \mathrm{ppm}$; MS $m / z$ (\%): $447\left(\mathrm{M}^{+}, 42\right), 431$ (47), 342 (36), 241 (19), 108 (46), 80 (100), 64 (92). Anal. calcd for $\mathrm{C}_{23} \mathrm{H}_{21} \mathrm{~N}_{5} \mathrm{O}_{3} \mathrm{~S}$ (447.51): C, 61.73; H, 4.73; N, 15.65. Found: C, 61.63; H, 4.59; N, $15.60 \%$

\section{3-(1-(2-(5-((4-Chlorophenyl)}

diazenyl)-4-methylthiazol-2-yl)hydrazono) ethyl)-6-methyl-2H-chromen-2-one (6e)

Red solid, m.p. $197^{\circ} \mathrm{C}-199^{\circ} \mathrm{C}$; IR $(\mathrm{KBr}) v \mathrm{~cm}^{-1}: 3,419(\mathrm{NH})$, $1,726(\mathrm{C}=\mathrm{O}), 1,608(\mathrm{C}=\mathrm{N}) ;{ }^{1} \mathrm{H}-\mathrm{NMR}\left(\mathrm{DMSO}-d_{6}\right) \delta: 2.13(\mathrm{~s}$, $\left.3 \mathrm{H}, \mathrm{CH}_{3}\right), 2.39\left(\mathrm{~s}, 3 \mathrm{H}, \mathrm{CH}_{3}\right), 2.74\left(\mathrm{~s}, 3 \mathrm{H}, \mathrm{CH}_{3}\right), 6.65-7.88(\mathrm{~m}$, $7 \mathrm{H}, \mathrm{Ar}-\mathrm{H}), 8.29(\mathrm{~s}, 1 \mathrm{H}$, coumarin- $\mathrm{H} 4), 11.08(\mathrm{~s}, \mathrm{br}, 1 \mathrm{H}, \mathrm{NH})$ ppm; MS m/z (\%): $451\left(\mathrm{M}^{+}, 73\right), 395$ (40), 214 (18), 127 (97), 80 (91), 64 (100). Anal. calcd for $\mathrm{C}_{22} \mathrm{H}_{18} \mathrm{ClN}_{5} \mathrm{O}_{2} \mathrm{~S}$ (451.93): C, 58.47; H, 4.01; N, 15.50. Found: C, 58.36; H, 3.84; N, 15.42\%.

\section{3-(1-(2-(5-((4-Bromophenyl)}

diazenyl)-4-methylthiazol-2-yl)hydrazono)

ethyl)-6-methyl-2H-chromen-2-one (6f)

Orange solid, m.p. $170^{\circ} \mathrm{C}-173^{\circ} \mathrm{C}$; IR $(\mathrm{KBr}) v \mathrm{~cm}^{-1}: 3,422$ $(\mathrm{NH}), 1,723(\mathrm{C}=\mathrm{O}), 1,601(\mathrm{C}=\mathrm{N}) ;{ }^{1} \mathrm{H}-\mathrm{NMR}\left(\mathrm{DMSO}-d_{6}\right) \delta$ : $2.12\left(\mathrm{~s}, 3 \mathrm{H}, \mathrm{CH}_{3}\right), 2.39\left(\mathrm{~s}, 3 \mathrm{H}, \mathrm{CH}_{3}\right), 2.74\left(\mathrm{~s}, 3 \mathrm{H}, \mathrm{CH}_{3}\right)$, 6.67-7.79 (m, 7H, Ar-H), $8.29(\mathrm{~s}, 1 \mathrm{H}$, coumarin-H4), 10.68 (s, br, 1H, NH) ppm; MS m/z (\%): $496\left(\mathrm{M}^{+}, 49\right), 377$ (53), 214 (27), 171 (48), 92 (86), 65 (100). Anal. calcd for $\mathrm{C}_{22} \mathrm{H}_{18} \mathrm{BrN}_{5} \mathrm{O}_{2} \mathrm{~S}$ (496.38): C, 53.23; $\mathrm{H}, 3.66 ; \mathrm{N}, 14.11$. Found: C, 53.04; H, 3.49; N, 14.02\%.

TABLE 2 | Optimization of the reaction conditions (catalyst loading, solvent, reaction time, and temperature) for the synthesis of compound $6 \mathbf{a}$.

\begin{tabular}{|c|c|c|c|c|c|}
\hline Entry & Catalyst (mol\%) & Solvent & Time (min) & Temperature $\left({ }^{\circ} \mathrm{C}\right)$ & Yield (\%) \\
\hline 1 & 1 & Dioxane & 25 & 50 & 57 \\
\hline 2 & 5 & Dioxane & 25 & 50 & 79 \\
\hline $3^{a}$ & 10 & Dioxane & 25 & 50 & 93 \\
\hline 5 & 10 & DMSO & 25 & 50 & 88 \\
\hline 6 & 10 & Dioxane & 20 & 50 & 89 \\
\hline 7 & 10 & Dioxane & 30 & 50 & 93 \\
\hline 10 & 10 & Dioxane & 25 & 60 & 93 \\
\hline
\end{tabular}

${ }^{a}$ The best reaction condition for the synthesis of compound $\mathbf{6 a}$ 


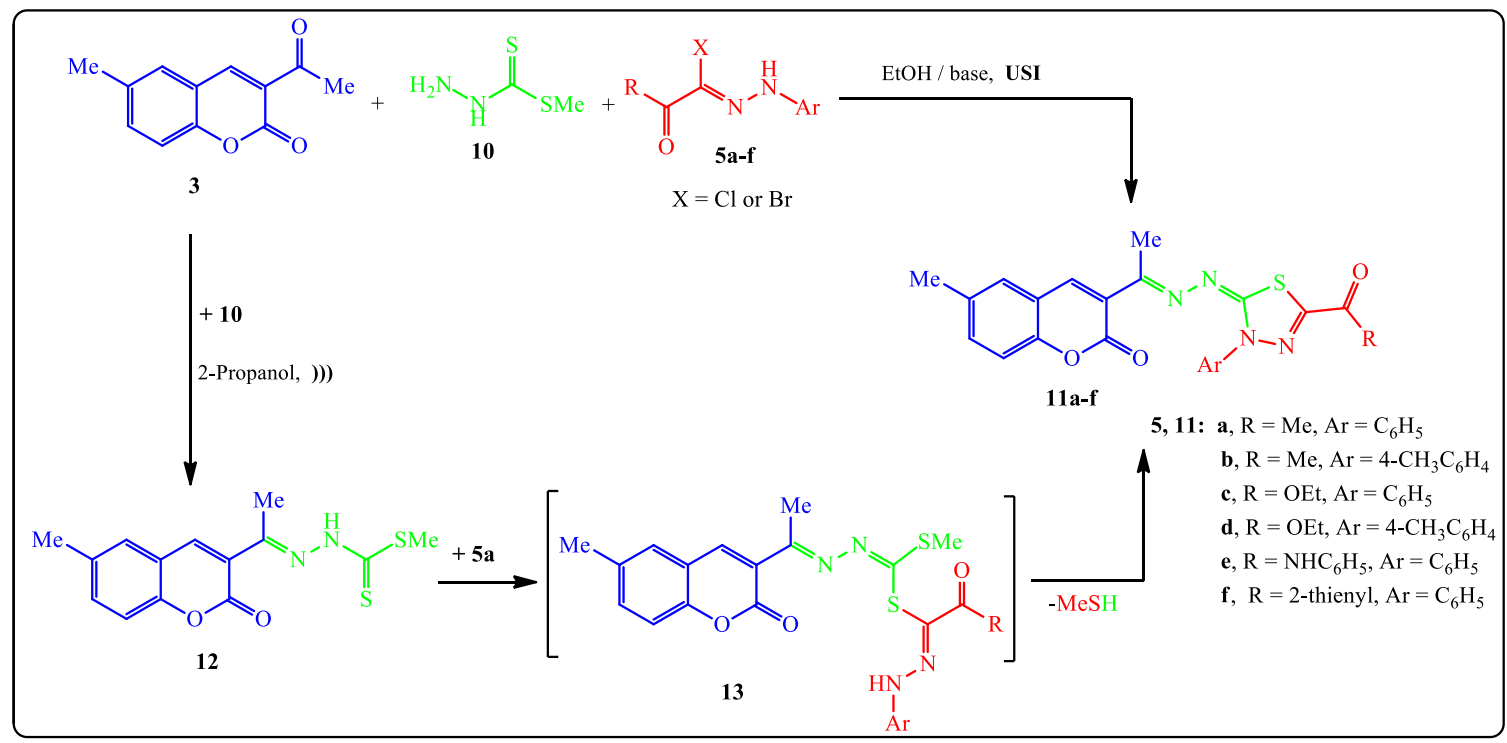

SCHEME 3 | Synthesis of thiadiazole derivatives 11a-f.

\section{6-Methyl-3-(1-(2-(4-methyl-5-((4-nitrophenyl)} diazenyl)thiazol-2-yl)hydrazono) ethyl)-2H-chromen-2-one $(6 \mathrm{~g})$

Brown solid, m.p. $213^{\circ} \mathrm{C}-215^{\circ} \mathrm{C}$; IR (KBr) $v \mathrm{~cm}^{-1}$ : 3,425 (NH), $1,729(\mathrm{C}=\mathrm{O}), 1,603(\mathrm{C}=\mathrm{N}) ;{ }^{1} \mathrm{H}-\mathrm{NMR}$ (DMSO- $\left.d_{6}\right) \delta: 2.25(\mathrm{~s}, 3 \mathrm{H}$, $\left.\mathrm{CH}_{3}\right), 2.45\left(\mathrm{~s}, 3 \mathrm{H}, \mathrm{CH}_{3}\right), 2.63\left(\mathrm{~s}, 3 \mathrm{H}, \mathrm{CH}_{3}\right), 6.65-8.21(\mathrm{~m}, 7 \mathrm{H}$, Ar-H), 8.30 (s, 1H, coumarin-H4), 11.09 (s, br, 1H, NH) ppm; MS $m / z$ (\%): $462\left(\mathrm{M}^{+}, 32\right), 375$ (27), 214 (100), 138 (37), 108 (39),
65 (73). Anal. calcd for $\mathrm{C}_{22} \mathrm{H}_{18} \mathrm{~N}_{6} \mathrm{O}_{4} \mathrm{~S}$ (462.48): C, 57.14; $\mathrm{H}, 3.92$ N, 18.17. Found: C, 57.04; H, 3.83; N, 18.03\%.

3-(1-(2-(5-((2,4-Dichlorophenyl) diazenyl)-4-methylthiazol-2-yl)hydrazono) ethyl)-6-methyl-2H-chromen-2-one (6h)

Red solid, m.p. $191^{\circ} \mathrm{C}-193^{\circ} \mathrm{C}$; IR (KBr) $v \mathrm{~cm}^{-1}: 3,426(\mathrm{NH})$, $1,725(\mathrm{C}=\mathrm{O}), 1,604(\mathrm{C}=\mathrm{N}) ;{ }^{1} \mathrm{H}-\mathrm{NMR}\left(\mathrm{DMSO}-d_{6}\right) \delta: 2.13(\mathrm{~s}$

TABLE 3 | Comparison of synthesis of thiadiazolylcoumarines 11a-f under USI using different basic catalysts on the time of reaction and the yield\% of the products.

Compound no.

\begin{tabular}{lr}
\cline { 2 - 2 } & Time (m) \\
\hline $\mathbf{1 1 a}$ & 50 \\
$\mathbf{1 1 b}$ & 50 \\
$\mathbf{1 1 c}$ & 56 \\
$\mathbf{1 1 d}$ & 53 \\
$\mathbf{1 1 e}$ & 52 \\
$\mathbf{1 1 f}$ & 60
\end{tabular}

TEA

(\%) Yield

72
74
73
72
72
72

Chitosan

\begin{tabular}{cc}
\hline Time (min) & (\%) Yield \\
41 & 79 \\
43 & 83 \\
46 & 84 \\
43 & 84 \\
36 & 86 \\
38 & 83
\end{tabular}

\section{g-Chitosan}

$\overline{\text { Time (min) } \quad \text { (\%) Yield }}$

Note. USI, ultrasonic irradiation; TEA, trimethylamine; g-chitosan, chitosan-grafted poly(viny/pyridine).

TABLE 4 | Optimization of the reaction conditions (solvent, reaction time, and temperature) for the synthesis of compound 11a.

\begin{tabular}{|c|c|c|c|c|c|}
\hline Entry & Catalyst (mol\%) & Solvent & Time (min) & Temperature $\left({ }^{\circ} \mathrm{C}\right)$ & Yield (\%) \\
\hline $1^{\mathrm{a}}$ & 10 & $\mathrm{EtOH}$ & 15 & 25 & 91 \\
\hline 2 & 10 & Dioxane & 15 & 25 & 81 \\
\hline 3 & 10 & DMSO & 15 & 25 & 83 \\
\hline 5 & 10 & $\mathrm{EtOH}$ & 20 & 25 & 91 \\
\hline 6 & 10 & $\mathrm{EtOH}$ & 15 & 50 & 91 \\
\hline
\end{tabular}

${ }^{a}$ The best reaction condition for the synthesis of compound 11a. 
TABLE 5 | Cytotoxic activities of tested compounds against HEPG2-1.

\begin{tabular}{llcc} 
Compd No & \multicolumn{1}{c}{$\mathbf{A r}$} & $\mathbf{I C _ { 5 0 }}(\boldsymbol{\mu M})$ \\
\hline Doxorubicin & - & - & $0.31 \pm 0.48$ \\
$\mathbf{6 a}$ & $\mathrm{Me}$ & $\mathrm{C}_{6} \mathrm{H}_{5}$ & $4.71 \pm 0.72$ \\
$\mathbf{6 b}$ & $\mathrm{Me}$ & $4-\mathrm{MeC}_{6} \mathrm{H}_{4}$ & $0.43 \pm 0.66$ \\
$\mathbf{6 c}$ & $\mathrm{Me}$ & $3-\mathrm{MeC}_{6} \mathrm{H}_{4}$ & $2.62 \pm 0.75$ \\
$\mathbf{6 d}$ & $\mathrm{Me}$ & $4-\mathrm{MeOC}_{6} \mathrm{H}_{4}$ & $0.29 \pm 0.45$ \\
$\mathbf{6 e}$ & $\mathrm{Me}$ & $4-\mathrm{ClC}_{6} \mathrm{H}_{4}$ & $5.79 \pm 0.81$ \\
$\mathbf{6 f}$ & $\mathrm{Me}$ & $4-\mathrm{BrC}_{6} \mathrm{H}_{4}$ & $5.03 \pm 0.56$ \\
$\mathbf{6 g}$ & $\mathrm{Me}$ & $4-\mathrm{NO}_{2} \mathrm{C}_{6} \mathrm{H}_{4}$ & $21.82 \pm 0.79$ \\
$\mathbf{6 h}$ & $\mathrm{Me}$ & $2,4-\mathrm{Cl}_{2} \mathrm{C}_{6} \mathrm{H}_{3}$ & $19.20 \pm 0.91$ \\
$\mathbf{6 i}$ & $2-\mathrm{Thienyl}$ & $\mathrm{C}_{6} \mathrm{H}_{5}$ & $11.37 \pm 0.49$ \\
$\mathbf{1 1 a}$ & $\mathrm{Me}$ & $\mathrm{C}_{6} \mathrm{H}_{5}$ & $7.06 \pm 0.77$ \\
$\mathbf{1 1 b}$ & $\mathrm{Me}$ & $4-\mathrm{MeC}_{6} \mathrm{H}_{4}$ & $5.28 \pm 0.73$ \\
$\mathbf{1 1 c}$ & $\mathrm{OEt}$ & $\mathrm{C}_{6} \mathrm{H}_{5}$ & $3.27 \pm 0.48$ \\
$\mathbf{1 1 d}$ & $\mathrm{OEt}$ & $4-\mathrm{MeC}_{6} \mathrm{H}_{4}$ & $1.73 \pm 0.61$ \\
$\mathbf{1 1 e}$ & $\mathrm{NHC} \mathrm{H}_{5}$ & $\mathrm{C}_{6} \mathrm{H}_{5}$ & $0.49 \pm 0.38$ \\
$\mathbf{1 1 f}$ & $2-\mathrm{Thienyl}$ & $\mathrm{C}_{6} \mathrm{H}_{5}$ & $14.23 \pm 0.59$
\end{tabular}

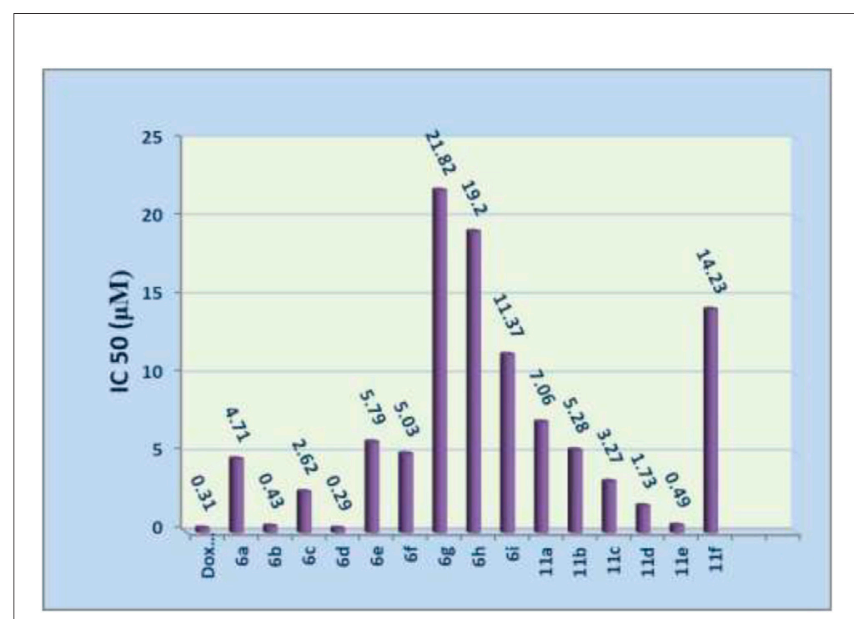

FIGURE 3 | Cytotoxic activities of tested compounds against HEPG2-1.

$\left.3 \mathrm{H}, \mathrm{CH}_{3}\right), 2.38\left(\mathrm{~s}, 3 \mathrm{H}, \mathrm{CH}_{3}\right), 2.73\left(\mathrm{~s}, 3 \mathrm{H}, \mathrm{CH}_{3}\right), 6.64-7.79(\mathrm{~m}$, $6 \mathrm{H}, \mathrm{Ar}-\mathrm{H}), 8.29(\mathrm{~s}, 1 \mathrm{H}$, coumarin-H4), $10.73(\mathrm{~s}, \mathrm{br}, 1 \mathrm{H}, \mathrm{NH})$ ppm; MS m/z (\%): $486\left(\mathrm{M}^{+}, 73\right), 451$ (60), 357 (48), 214 (29), 161 (85), 80 (71), 64 (100). Anal. calcd for $\mathrm{C}_{22} \mathrm{H}_{17} \mathrm{Cl}_{2} \mathrm{~N}_{5} \mathrm{O}_{2} \mathrm{~S}$ (486.37): C, 54.33; H, 3.52; N, 14.40. Found: C, 54.27; H, 3.50; $\mathrm{N}, 14.27 \%$.

6-Methyl-3-(1-(2-(5-(phenyldiazenyl)-4-(thiophen-2-yl) thiazol-2-yl)hydrazono)ethyl)-2H-chromen-2-one (6i) Orange solid, m.p. $166^{\circ} \mathrm{C}-168^{\circ} \mathrm{C}$; IR $(\mathrm{KBr}) v \mathrm{~cm}^{-1}: 3,420(\mathrm{NH})$, 1,727 $(\mathrm{C}=\mathrm{O}), 1,605(\mathrm{C}=\mathrm{N}) ;{ }^{1} \mathrm{H}-\mathrm{NMR}$ (DMSO- $\left.d_{6}\right) \delta: 2.18(\mathrm{~s}, 3 \mathrm{H}$, $\left.\mathrm{CH}_{3}\right), 2.39$ (s, 3H, $\left.\mathrm{CH}_{3}\right), 6.68-8.06(\mathrm{~m}, 11 \mathrm{H}, \mathrm{Ar}-\mathrm{H}), 8.39$ (s, $1 \mathrm{H}$, coumarin-H4), 11.50 (s, br, $1 \mathrm{H}, \mathrm{NH}) \mathrm{ppm}$; MS m/z (\%): $485\left(\mathrm{M}^{+}\right.$, 27), 456 (83), 383 (69), 214 (18), 135 (14), 111 (100), 77 (79). Anal. calcd for $\mathrm{C}_{25} \mathrm{H}_{19} \mathrm{~N}_{5} \mathrm{O}_{2} \mathrm{~S}_{2}$ (485.58): C, 61.84; H, 3.94; N, 14.42. Found: C, 61.71; H, 3.88; N, $14.36 \%$.

\section{Alternate Synthesis of $\mathbf{6 a}$}

i) Synthesis of 2-(1-(6-methyl-2-oxo-2H-chromen-3-yl) ethylidene)hydrazine-1-carbothioamide (7). A catalytic amount of concentrated hydrochloric acid was added to a mixture of 3-acetyl-6-methyl-2H-chromen-2-one (3) (2.02 g, $10 \mathrm{mmol})$ and thiosemicarbazide $4(0.91 \mathrm{~g}, 10 \mathrm{mmol})$ in $50 \mathrm{ml}$ of ethanol. The reaction mixture was irradiated by an ultrasonic generator in a water bath at $50^{\circ} \mathrm{C}$ for $30 \mathrm{~min}$. The precipitate that formed after cooling was filtered, washed with ethanol, and recrystallized from acetic acid to give a pure product of compound 7 as yellowish-white solid in $72 \%$ yield; m.p. $231^{\circ} \mathrm{C}-233^{\circ} \mathrm{C}$; IR (KBr) $v \mathrm{~cm}^{-1}: 3,431,4,237,3,159(\mathrm{NH}$ and $\left.\mathrm{NH}_{2}\right), 1,726(\mathrm{C}=\mathrm{O}), 1,604(\mathrm{C}=\mathrm{N}) ;{ }^{1} \mathrm{H}-\mathrm{NMR}\left(\mathrm{DMSO}-d_{6}\right) \delta$ : 2.25 (s, 3H, $\left.\mathrm{CH}_{3}\right), 2.37$ (s, 3H, $\left.\mathrm{CH}_{3}\right), 7.30-7.53$ (m, 3H, Ar-H), 7.92 (s, 1H, coumarin-H4), 8.37 (s, br, 2H, $\mathrm{NH}_{2}$ ), 10.40 (s, br, $1 \mathrm{H}, \mathrm{NH}) \mathrm{ppm}$; MS m/z (\%): $275\left(\mathrm{M}^{+}, 63\right), 214$ (49), 175 (29), 111 (100), 77 (69), 63 (82). Anal. calcd for $\mathrm{C}_{13} \mathrm{H}_{13} \mathrm{~N}_{3} \mathrm{O}_{2} \mathrm{~S}$ (275.33): C, 56.71; H, 4.76; N, 15.26. Found: C, 56.58; H, $4.71 ; \mathrm{N}, 15.09 \%$.

\section{Reaction of thiosemicarbazone 7 with 2-oxo- $\mathrm{N}$-phenylpropanehydrazonoyl chloride (5a)}

Chitosan-grafted poly(vinylpyridine) $(0.1 \mathrm{~g})$ was added to a stirred mixture of thiosemicarbazone $7(0.275 \mathrm{~g}, 1 \mathrm{mmol})$ and 2-oxo- $N$-phenylpropanehydrazonoyl chloride (5a) (0.196 g, $1 \mathrm{mmol})$ in dioxane $(15 \mathrm{ml})$. The reaction mixture was irradiated by an ultrasonic generator in a water bath at $40^{\circ} \mathrm{C}$ for $30 \mathrm{~min}$. The hot solution was filtered to remove g-chitosan and excess solvent was removed under reduced pressure. The reaction mixture was triturated with methanol, and the product separated was filtered, washed with methanol, dried, and recrystallized from DMF to give the corresponding product $\mathbf{6 a}$, which was identical in all aspects (m.p., mixed m.p., and IR spectra) with those obtained from the one-pot synthesis of $3+$ $4+5 a$.

\section{General Procedure for Synthesis of 1,3,4-Thiadiazole Derivatives 11a-f}

Method A: A mixture of 3-acetyl-6-methyl-2H-chromen-2-one (3) $(2.02 \mathrm{~g}, 10 \mathrm{mmol})$, methyl hydrazinecarbodithioate (10) $(0.122 \mathrm{~g}, 1 \mathrm{mmol})$, and the appropriate hydrazonoyl halides $5 \mathbf{a}-\mathbf{f}(1 \mathrm{mmol})$ in ethanol $(20 \mathrm{ml})$ containing TEA $(0.07 \mathrm{ml})$ was stirred at room temperature for $4-8 \mathrm{~h}$. The resulting solid was collected and recrystallized from DMF to give the corresponding 1,3,4-thiadiazoles 11a-f. Products 11a-f together with their physical constants are listed below.

Method B: A mixture of equimolar amounts of 3, 10, and the appropriate $5 \mathbf{a}-\mathbf{f}(1 \mathrm{mmol}$ each) in ethanol $(20 \mathrm{ml})$ containing chitosan $(0.1 \mathrm{~g})$ was irradiated by an ultrasonic generator in a water bath at $25^{\circ} \mathrm{C}$ for $10-30 \mathrm{~min}$ (monitored by TLC). The hot solution was filtered to remove chitosan, and excess solvent was removed under reduced pressure. The reaction mixture was triturated with methanol, and the product separated was filtered, washed with methanol, 
dried, and recrystallized from DMF to give compounds 11a-f.

Method C: The same procedure in method B using grafted chitosan $(0.1 \mathrm{~g})$ instead of chitosan.

\section{3-(1-)(5-Acetyl-}

3-phenyl-1,3,4-thiadiazol-2(3H)-ylidene)hydrazono) ethyl)-6-methyl-2H-chromen-2-one (11a)

Yellow solid, m.p. $214^{\circ} \mathrm{C}-216^{\circ} \mathrm{C}$; IR (KBr) $v \mathrm{~cm}^{-1}: 1,726,1,696$ $(2 \mathrm{C}=\mathrm{O}), 1,602(\mathrm{C}=\mathrm{N}) ;{ }^{1} \mathrm{H}-\mathrm{NMR}\left(\mathrm{DMSO}-d_{6}\right) \delta: 2.37(\mathrm{~s}, 3 \mathrm{H}$, $\left.\mathrm{CH}_{3}\right), 2.44\left(\mathrm{~s}, 3 \mathrm{H}, \mathrm{CH}_{3}\right), 2.57\left(\mathrm{~s}, 3 \mathrm{H}, \mathrm{CH}_{3}\right), 7.34-7.72(\mathrm{~m}, 8 \mathrm{H}$, $\mathrm{Ar}-\mathrm{H}$ ), 8.56 (s, $1 \mathrm{H}$, coumarin-H4) ppm; ${ }^{13} \mathrm{C}-\mathrm{NMR}$ (DMSO$\left.d_{6}\right): \delta 14.7,20.1,29.9\left(\mathrm{CH}_{3}\right), 107.6,115.8,120.4,125.5,127.3$, $127.7,128.7,129.5,130.1,134.1,135.3,140.0,146.8,152.7$ (Ar-C), 168.5, $195.0(\mathrm{C}=\mathrm{O}) \mathrm{ppm}$; MS $m / z(\%): 418\left(\mathrm{M}^{+}, 42\right)$, 338 (53), 214 (4), 177 (51), 90 (62), 64 (100). Anal. calcd for $\mathrm{C}_{22} \mathrm{H}_{18} \mathrm{~N}_{4} \mathrm{O}_{3} \mathrm{~S}$ (418.47): C, 63.14; H, 4.34; N, 13.39. Found: $\mathrm{C}$, $63.05 ; \mathrm{H}, 4.31 ; \mathrm{N}, 13.26 \%$.

\section{3-(1-((5-Acetyl-3- \\ (p-tolyl)-1,3,4-thiadiazol-2(3H)-ylidene)hydrazono) ethyl)-6-methyl-2H-chromen-2-one (11b)}

Yellow solid, m.p. $201^{\circ} \mathrm{C}-203^{\circ} \mathrm{C}$; IR (KBr) $v \mathrm{~cm}^{-1}: 1,724,1,699$ $(2 \mathrm{C}=\mathrm{O}), 1,606(\mathrm{C}=\mathrm{N}) ;{ }^{1} \mathrm{H}-\mathrm{NMR}\left(\mathrm{DMSO}-d_{6}\right) \delta: 2.25\left(\mathrm{~s}, 3 \mathrm{H}, \mathrm{CH}_{3}\right)$, 2.37 (s, 3H, $\left.\mathrm{CH}_{3}\right), 2.41$ (s, 3H, $\left.\mathrm{CH}_{3}\right), 2.58\left(\mathrm{~s}, 3 \mathrm{H}, \mathrm{CH}_{3}\right), 7.31-7.63$ (m, 6H, Ar-H), 7.73 (s, 1H, Ar-H), 8.57 (s, 1H, coumarin-H4) ppm; MS m/z (\%): 432 (M $\left.{ }^{+}, 68\right), 331$ (60), 186 (44), 158 (41), 91 (100), 77 (70). Anal. calcd for $\mathrm{C}_{23} \mathrm{H}_{20} \mathrm{~N}_{4} \mathrm{O}_{3} \mathrm{~S}$ (432.50): C, 63.87; H, 4.66; N, 12.95. Found: C, 63.71; H, 4.62; N, 12.84\%.

\section{Ethyl 5-((1-(6-methyl-2-oxo-2H-chromen-3-yl) ethylidene)hydrazono)-4-phenyl- 4,5-dihydro-1,3,4-thiadiazole-2-carboxylate (11c)}

Yellow solid, m.p. $190^{\circ} \mathrm{C}-192^{\circ} \mathrm{C}$; IR $(\mathrm{KBr}) v \mathrm{~cm}^{-1}: 1,740,1,722$ $(2 \mathrm{C}=\mathrm{O}), 1,603(\mathrm{C}=\mathrm{N}) ;{ }^{1} \mathrm{H}-\mathrm{NMR}\left(\mathrm{DMSO}-d_{6}\right) \delta: 1.30(\mathrm{t}, 3 \mathrm{H}, J=6.9$ $\mathrm{Hz}, \mathrm{CH}_{2} \mathrm{CH}_{3}$ ), 2.37 (s, 3H, $\left.\mathrm{CH}_{3}\right), 2.62\left(\mathrm{~s}, 3 \mathrm{H}, \mathrm{CH}_{3}\right), 4.27$ (q, $2 \mathrm{H}$, $\left.J=6.9 \mathrm{~Hz}, \mathrm{CH}_{2} \mathrm{CH}_{3}\right), 7.22-7.69(\mathrm{~m}, 7 \mathrm{H}, \mathrm{Ar}-\mathrm{H}), 7.73(\mathrm{~s}, 1 \mathrm{H}$, $\mathrm{Ar}-\mathrm{H}), 8.55$ (s, $1 \mathrm{H}$, coumarin-H4) ppm; MS m/z (\%): $448\left(\mathrm{M}^{+}\right.$, 26), 338 (39), 214 (63), 158 (50), 90 (86), 77 (100). Anal. calcd for $\mathrm{C}_{23} \mathrm{H}_{20} \mathrm{~N}_{4} \mathrm{O}_{4} \mathrm{~S}$ (448.50): C, 61.60; H, 4.50; N, 12.49. Found: C, 61.52; H, 4.38; N, 12.36\%.

\section{Ethyl 5-((1-(6-methyl-2-oxo-2H-chromen-3-yl) ethylidene)hydrazono)-4-( $p$ - tolyl)-4,5-dihydro-1,3,4-thiadiazole-2-carboxylate (11d)}

Yellow solid, m.p. $171^{\circ} \mathrm{C}-173^{\circ} \mathrm{C}$; IR $(\mathrm{KBr}) v \mathrm{~cm}^{-1}: 1,742,1,722$ $(2 \mathrm{C}=\mathrm{O}), 1,606(\mathrm{C}=\mathrm{N}) ;{ }^{1} \mathrm{H}-\mathrm{NMR}\left(\mathrm{DMSO}-d_{6}\right) \delta: 1.27(\mathrm{t}, 3 \mathrm{H}, J=6.9$ $\mathrm{Hz}, \mathrm{CH}_{2} \mathrm{CH}_{3}$ ), 2.23 (s, 3H, $\left.\mathrm{CH}_{3}\right), 2.37$ (s, 3H, $\left.\mathrm{CH}_{3}\right), 2.65$ (s, 3H, $\left.\mathrm{CH}_{3}\right), 4.29\left(\mathrm{q}, 2 \mathrm{H}, J=6.9 \mathrm{~Hz}, \mathrm{CH}_{2} \mathrm{CH}_{3}\right), 7.33-7.56(\mathrm{~m}, 6 \mathrm{H}$, Ar-H), 7.71 (s, 1H, Ar-H), 8.55 (s, 1H, coumarin-H4) ppm; MS $\mathrm{m} / z$ (\%): 462 ( $\left.\mathrm{M}^{+}, 70\right), 384$ (94), 331 (40), 186 (36), 91 (100), 77 (69). Anal. calcd for $\mathrm{C}_{24} \mathrm{H}_{22} \mathrm{~N}_{4} \mathrm{O}_{4} \mathrm{~S}$ (462.52): C, 62.32; $\mathrm{H}, 4.79 ; \mathrm{N}$, 12.11. Found: C, 62.19; H, 4.64; N, 12.03\%.
5-((1-(6-Methyl-2-oxo-2H-chromen-3-yl)ethylidene) hydrazono)- $N$,4-diphenyl-4,5-

dihydro-1,3,4-thiadiazole-2-carboxamide (11e)

Yellow solid, m.p. $190^{\circ} \mathrm{C}-192^{\circ} \mathrm{C}$; IR (KBr) $v \mathrm{~cm}^{-1}: 3,427(\mathrm{NH})$, $1,740,1,679(2 \mathrm{C}=\mathrm{O}), 1,600(\mathrm{C}=\mathrm{N}) ;{ }^{1} \mathrm{H}-\mathrm{NMR}\left(\mathrm{DMSO}-d_{6}\right) \delta$ : $2.37\left(\mathrm{~s}, 3 \mathrm{H}, \mathrm{CH}_{3}\right), 2.57\left(\mathrm{~s}, 3 \mathrm{H}, \mathrm{CH}_{3}\right), 7.34-7.57(\mathrm{~m}, 12 \mathrm{H}$, Ar-H), 7.72 (s, 1H, Ar-H), $8.56(\mathrm{~s}, 1 \mathrm{H}$, coumarin-H4), 10.63 (s, br, 1H, NH) ppm; MS m/z (\%): $495\left(\mathrm{M}^{+}, 49\right), 418(37), 331$ (41), 186 (54), 91 (100), 77 (86), 64 (61). Anal. calcd for $\mathrm{C}_{27} \mathrm{H}_{21} \mathrm{~N}_{5} \mathrm{O}_{3} \mathrm{~S}$ (495.56): C, 65.44; H, 4.27; N, 14.13. Found: C, 65.28; H, 4.21; N, 14.05\%.

\section{6-Methyl-3-(1-((3-phenyl-5-(thiophene-2-carbonyl)- 1,3,4-thiadiazol-2(3H)-ylidene)hydrazono) ethyl)-2H-chromen-2-one (11f)}

Yellow solid, m.p. $240^{\circ} \mathrm{C}-242^{\circ} \mathrm{C}$; IR (KBr) $v \mathrm{~cm}^{-1}: 1,726,1,693$ $(2 \mathrm{C}=\mathrm{O}), 1,607(\mathrm{C}=\mathrm{N}) ;{ }^{1} \mathrm{H}-\mathrm{NMR}\left(\mathrm{DMSO}-d_{6}\right) \delta: 2.37\left(\mathrm{~s}, 3 \mathrm{H}, \mathrm{CH}_{3}\right)$, $2.64\left(\mathrm{~s}, 3 \mathrm{H}, \mathrm{CH}_{3}\right), 7.34-7.69(\mathrm{~m}, 10 \mathrm{H}, \mathrm{Ar}-\mathrm{H}), 7.71(\mathrm{~s}, 1 \mathrm{H}, \mathrm{Ar}-\mathrm{H})$, 8.55 (s, $1 \mathrm{H}$, coumarin-H4) ppm; MS m/z (\%): $486\left(\mathrm{M}^{+}, 27\right), 426$ (60), 330 (39), 111 (51), 80 (100), 64 (89). Anal. calcd for $\mathrm{C}_{25} \mathrm{H}_{18} \mathrm{~N}_{4} \mathrm{O}_{3} \mathrm{~S}_{2}$ (486.56): C, 61.71; H, 3.73; N, 11.52. Found: C, $61.58 ; \mathrm{H}, 3.64 ; \mathrm{N}, 11.41 \%$.

\section{Alternate Synthesis of 11a}

i) Synthesis of methyl 2-(1-(6-methyl-2-oxo-2H-chromen-3-yl) ethylidene)hydrazine-1-carbodithioate (12). To a solution of 3-acetyl-6-methyl-2H-chromen-2-one (3) (2.02 g, $10 \mathrm{mmol})$ in 2-propanol $(20 \mathrm{ml})$, methyl hydrazinecarbodithioate (10) (1.22 g, $10 \mathrm{mmol}$ ) was added. The reaction mixture was irradiated by an ultrasonic generator in a water bath at $30^{\circ} \mathrm{C}$ for $30 \mathrm{~min}$. The precipitate that formed after cooling was filtered, washed with ethanol, and recrystallized from acetic acid to give pure product of compound 12 as yellowishwhite solid in $68 \%$ yield; m.p. $175^{\circ} \mathrm{C}-177^{\circ} \mathrm{C}$; IR $(\mathrm{KBr}) v \mathrm{~cm}^{-1}$ : $3,420(\mathrm{NH}), 1,723(\mathrm{C}=\mathrm{O}), 1,604(\mathrm{C}=\mathrm{N}){ }^{1} \mathrm{H}-\mathrm{NMR}\left(\mathrm{DMSO}-d_{6}\right)$ $\delta: 2.25\left(\mathrm{~s}, 3 \mathrm{H}, \mathrm{CH}_{3}\right), 2.37\left(\mathrm{~s}, 3 \mathrm{H}, \mathrm{CH}_{3}\right), 2.57\left(\mathrm{~s}, 3 \mathrm{H}, \mathrm{SCH}_{3}\right), 7.33$ $(\mathrm{d}, J=9 \mathrm{~Hz}, 1 \mathrm{H}, \mathrm{Ar}-\mathrm{H}), 7.54(\mathrm{~d}, J=9 \mathrm{~Hz}, 1 \mathrm{H}, \mathrm{Ar}-\mathrm{H}), 7.71(\mathrm{~s}$, $1 \mathrm{H}, \mathrm{Ar}-\mathrm{H}), 8.55$ (s, 1H, coumarin-H4), 11.79 (s, br, 1H, NH) ppm; MS m/z (\%): $306\left(\mathrm{M}^{+}, 18\right), 186$ (40), 158 (37), 128 (39), 90 (100), 77 (86), 64 (75). Anal. calcd for $\mathrm{C}_{14} \mathrm{H}_{14} \mathrm{~N}_{2} \mathrm{O}_{2} \mathrm{~S}_{2}$ (306.40): C, 54.88; H, 4.61; N, 9.14. Found: C, 54.70; H, 4.58; $\mathrm{N}, 9.06 \%$.

ii) Reaction of carbodithioate 12 with 2-oxo$N$-phenylpropanehydrazonoyl chloride (5a). TEA $(0.5 \mathrm{ml})$ was added to a stirred ethanolic solution of methyl hydrazinecarbodithioate (10) $(0.122 \mathrm{~g}, 1 \mathrm{mmol})$ and 2-oxo$N$-phenylpropanehydrazonoyl chloride (5a) (0.196 g, $1 \mathrm{mmol}$ ). The reaction mixture was irradiated by an ultrasonic generator in a water bath at $40^{\circ} \mathrm{C}$ for $30 \mathrm{~min}$. The solid precipitated after cooling and was filtered off, washed with water, dried, and finally recrystallized from DMF to give the corresponding product 11a, which was identical in all aspects (m.p., mixed m.p., and IR spectra) with those obtained from the one-pot synthesis of $\mathbf{3}+\mathbf{1 0}+\mathbf{5 a}$. 


\section{Anticancer Activities}

The cytotoxic evaluation of the synthesized compounds was carried out at the Regional Center for Mycology and Biotechnology at Al-Azhar University, Cairo, Egypt, according to the reported method (Gomha et al., 2015c; Gomha et al., 2015d). For more details, see the supporting information file.

\section{RESULTS AND DISCUSSION}

In continuation of our previous work to synthesize bioactive heterocyclic compounds under mild conditions, herein we wish to report a mild and efficient procedure for the synthesis of some thiazolyl-coumarins via the one-pot, threecomponent reaction of 3-acetyl-6-methyl-2H-chromen-2one (3) (prepared previously from the reaction of $\mathbf{1}$ with 2) (Scheme 1) (Toan et al., 2020), thiosemicarbazone (4), and the appropriate hydrazonoyl halides 5a-i [52] in dioxane under ultrasonic irradiation (USI) at $50^{\circ} \mathrm{C}$ for $20-60 \mathrm{~min}$, in the presence of different basic catalysts such as TEA, chitosan, and chitosan-grafted poly(vinylpyridine) (Scheme 1). The development of all reactions was tracked by TLC. At the outset, the identification of the best basic catalyst was examined (Table 1).

As shown from Table 1, chitosan-grafted poly(vinylpyridine) was the best choice of a basic catalyst under USI. The reaction proceeds smoothly with an electronrich as well as electron-deficient substituent on the aromatic benzene ring of hydrazonoyl halides 5 . The structures of isolated products $\mathbf{6 a - i}$ were evidenced by spectral data together with elemental analyses. We have observed that under the same reaction conditions, the yields of the desired products $\mathbf{6 a - i}$ increase by changing TEA into chitosan. Moreover, using grafted chitosan as a basic catalyst has a significant increasing effect on the product yields. In addition, the heating under USI was more efficient than conventional heating, as it reduced the reaction time and increased the product yields in the case of compounds $\mathbf{6 a}-\mathbf{c}$ as shown in Supplementary Table S1.

The elemental analyses and spectroscopic data of the obtained products $\mathbf{6 a - i}$ supported the assigned structures. The IR spectrum of $\mathbf{6 a}$ as a representative example exhibits two strong stretching frequencies in the regions of 3,427 and $1,724 \mathrm{~cm}^{-1}$, attributable to the $\mathrm{NH}$ and $\mathrm{C}=\mathrm{O}$ groups, respectively. Its ${ }^{1} \mathrm{H}$-NMR spectrum displayed five singlet signals for the $3 \mathrm{CH}_{3}$, coumarin- $\mathrm{H} 4$, and $\mathrm{NH}$ protons at $\delta$ $2.13,2.44,2.67,8.29$, and $11.16 \mathrm{ppm}$, in addition to the characteristic multiplet signal for the eight aromatic protons. Moreover, its ${ }^{13} \mathrm{C}-\mathrm{NMR}$ showed three aliphatic signals for the three methyl groups at $11.3,16.2$, and 20.1 ppm; 15 aromatic signals at $115.6-151.4 \mathrm{ppm}$; and one carbonyl signal at $168.1 \mathrm{ppm}$. IR $(\mathrm{KBr})$ spectra showed three bands at $v 3,422,1,671$, and $1,653 \mathrm{~cm}^{-1}$ assignable to the $\mathrm{NH}$ and $2 \mathrm{C}=\mathrm{O}$ groups. The mass spectrum is also an additional evidence for supporting the obtained structure, which gave a molecular ion at $\mathrm{m} / z 417.13\left[\mathrm{M}^{+}\right]$(Gomha et al., 2015b;
Gomha et al., 2015c; Alshabanah et al., 2021; Gomha et al., 2021).

On the other hand, reaction of 2-(1-(6-methyl-2-oxo2H-chromen-3-yl)ethylidene)hydrazine-1-carbothioamide (7) (prepared separately through condensation of 3-acetyl-6methyl-2H-chromen-2-one (3) with thiosemicarbazide in ethanol containing drops of concentrated $\mathrm{HCl}$ ) with 2-oxo$N$-phenylpropanehydrazonoyl chloride (5a) (Shawali and Gomha, 2000) gave an identical product in IR, m.p., and mixed m.p. with 6a (Scheme 2). The overall yield for this 2step reaction is $83 \%$.

To achieve the best experimental conditions and the different factors (such as catalyst loading, temperature, solvent, and reaction time) on the reaction of $\mathbf{3}+\mathbf{4}+\mathbf{5 a}$ in the presence of a catalytic amount of chitosan-grafted poly(vinylpyridine) in one-pot synthesis under USI to get 6-methyl-3-(1-(2-(4-methyl5-(phenyldiazenyl)thiazol-2-yl)hydrazono)ethyl)- $2 \mathrm{H}$-chromen-

2-one (6a), the following was carried out.

In the first step, we examined the effect of the amount of catalyst for the synthesis of compound 6a (Table 2, entries 1-3). The best results were obtained using $10 \mathrm{~mol} \%$ of catalyst (93\%) (Table 2, entry 3). Using lower amounts of catalyst resulted in lower yields (Table 2).

In the next step, the efficiency of the different solvents was examined under USI (Table 2, entries 3-5). Screening of various solvents showed that the formation of product $\mathbf{6 a}$ proceeded in the highest yield with a higher reaction rate in dioxane (Table 2, entry 3).

Furthermore, the reaction time was examined under USI (Table 2, entries 3, 6, and 7). The best time for the formation of product $\mathbf{6 a}$ was 25 min (Table 2, entry 3).

In continuation, the effect of temperatures was also tested on the reaction, and the results are presented in Table 2 (entries 3, 8, 9, and 10). According to Table 2, increasing the reaction temperature from $25^{\circ} \mathrm{C}$ to $40^{\circ} \mathrm{C}-60^{\circ} \mathrm{C}$ under USI increases the yields of products from $80 \%$ to $88 \%-93 \%$, respectively. Finally, $50^{\circ} \mathrm{C}$ was selected as the optimum temperature (Table 2, entry 3).

As shown in Table 2, we observed that the optimum reaction conditions for the formation of product $\mathbf{6 a}$ are as follows: reaction of $3+4+5 \mathbf{a}$ in dioxane under USI in the presence of $10 \mathrm{~mol} \%$ of chitosan-grafted poly(vinylpyridine) at $50^{\circ} \mathrm{C}$ for $25 \mathrm{~min}$.

Thus, irradiation of $\mathbf{3}+\mathbf{4}+\mathbf{5 b}-\mathbf{i}$ under the optimum conditions led to the formation of 6-methyl-3-(1-(2-(4substituted-5-(aryldiazenyl)thiazol-2-yl)hydrazono)ethyl)$2 \mathrm{H}$-chromen-2-one derivatives $\mathbf{6 b}-\mathbf{i}$ (Scheme $\mathbf{1}$ ).

Our research has now been expanded to include the synthesis of new 1,3,4-thiadiazole derivatives in an attempt to functionalize the target 3-thiadiazolyl coumarins 11a-f. Thus, treatment of compound 3, methyl hydrazinecarbodithioate (10), and various derivatives of hydrazonoyl halides $\mathbf{5 a}-\mathbf{f}$ in $\mathrm{EtOH}$ under USI at $25^{\circ} \mathrm{C}$ for $10-30 \mathrm{~min}$ in the presence of TEA or the chitosan or chitosan-grafted poly(vinylpyridine) as a basic catalyst afforded the respective 1,3,4-thiadiazoles 11a-f as depicted in Scheme 3.

Table 3 shows the yield \% of the isolated products 11a-f, the g-chitosan as a basic catalyst prevailed over chitosan and TEA under sonication technique. 
The structures of products 11a-f were elucidated based on spectral and analytical data as illustrated in the Methods and Methodology. For example, the IR spectra of the isolated products 11 revealed the existence of the characteristic bands for the two $\mathrm{C}=\mathrm{O}$ groups at the normal wave numbers. The ${ }^{1} \mathrm{H}-\mathrm{NMR}$ spectra of compound 11a showed the expected signals at $\delta: 2.37$, $2.44,2.57\left(3 \mathrm{~s}, 3 \mathrm{CH}_{3}\right), 8.56(\mathrm{~s}$, coumarin- $\mathrm{H} 4)$ in addition to one multiplet signal at $\delta 7.21-7.44 \mathrm{ppm}$ due to eight aromatic protons. ${ }^{13} \mathrm{C}-\mathrm{NMR}$ (DMSO- $d_{6}$ ) for compound 11a showed the characteristic signals for $3 \mathrm{CH}_{3}$ and the two $\mathrm{C}=\mathrm{O}$ groups at $\delta$ $14.7,20.1,29.9,168.5$, and $195.0 \mathrm{ppm}$, in addition to the expected aromatic carbons. The mass spectra of products $11 \mathbf{a}-\mathbf{f}$ revealed a molecular ion peak for each one, which is consistent with the respective molecular weight.

Alternatively, compound 11a was synthesized from a reaction of 2oxo- $\mathrm{N}$-phenylpropanehydrazonoyl chloride (5a) in $\mathrm{EtOH}$ containing a catalytic amount of g-chitosan under USI with carbothioamide 12 (prepared separately through condensation of compound 3 with methyl hydrazinecarbodithioate (10) in 2-propanol). The obtained product was found to be identical to 11a in all regards (m.p., TLC, and IR spectrum), which provides additional evidence to all 11a-f structures. The overall yield for this 2-step process was $78 \%$.

Also, one-pot synthesis of $\mathbf{3}+\mathbf{1 0}+\mathbf{5 a}$ under USI in the presence of a catalytic amount of chitosan-grafted poly(vinylpyridine) was examined at different temperatures, solvents, and reaction times. This reaction led to the formation of 1,3,4-thiadiazole 11a.

In the first step, we examined the effect of different solvents under USI (Table 4, entries 1-3). Screening of various solvents showed that the formation of product 11a proceeded in the highest yield with a higher reaction rate in ethanol (Table 4, entry 1).

We also examined the reaction time under USI (Table 4, entries 1, 4, and 5). The best time for the formation of product 11a is $15 \mathrm{~min}$ (Table 4, entry 1 ).

Finally, we also tested the reaction temperatures, and the results are presented in Table 4 (entries 1 and 6). According to Table 4, increasing the reaction temperature from $25^{\circ} \mathrm{C}$ to $50^{\circ} \mathrm{C}$ did not affect the yield of the product. Finally, $25^{\circ} \mathrm{C}$ was selected as the optimum temperature (Table 4 , entry 1 ).

As shown in Table 4, we can observe that the optimum reaction conditions for the formation of product 11a are as follows: reaction of $\mathbf{3}+\mathbf{1 0}+\mathbf{5 a}$ in ethanol under USI in the presence of $0.1 \mathrm{~g}$ of chitosan-grafted poly(vinylpyridine) at $25^{\circ} \mathrm{C}$ for $15 \mathrm{~min}$.

Thus, irradiation of $\mathbf{3}+\mathbf{1 0}+\mathbf{5 b}-\mathbf{i}$ under the optimum conditions led to formation of the respective 1,3,4-thiadiazole derivatives 11b-i (Scheme 3).

Generally, the formation of products $6 \mathbf{a}-\mathbf{i}$ and $11 \mathbf{a}-\mathbf{f}$ with the application of the USI/catalytic system in a three-component one-pot reaction gave high reaction yield in short reaction duration, and the achieved results showed the tremendous synergistic effect between g-chitosan and USI.

\section{Antitumor Activity}

The cytotoxic activity of the newly prepared compounds was determined against liver carcinoma cell line (HEPG2-1) using the 3-(4,5-dimethylthiazol-2-yl)-2,5-diphenyltetrazolium bromide (MTT) assay. Doxorubicin was used as a reference drug. Data generated were used to plot a dose-response curve of which the concentration $(\mu \mathrm{M})$ of the test compounds required to kill $50 \%$ of the cell population $\left(\mathrm{IC}_{50}\right)$ was determined. The cytotoxic activity was expressed as the mean $\mathrm{IC}_{50}$ of three independent experiments (Table 5 and Figure 3). The results revealed that the descending order of activity of the tested compounds was as follows: $6 \mathrm{~d}>\mathbf{6 b}>\mathbf{1 1 e}>11 \mathrm{1d}>\mathbf{6 c}>11 \mathrm{c}>\mathbf{6 a}>\mathbf{6 f}>11 \mathrm{~b}>\mathbf{6 e}>$ $11 \mathrm{a}>6 \mathrm{i}>11 \mathrm{f}>6 \mathrm{~h}>6 \mathrm{~g}$.

\section{Structure-Activity Relationship}

The activities of the synthesized compounds depend on the structural skeleton and electronic environment of the molecules.

Based on our limited study, the 1,3-thiazole derivatives have an in vitro inhibitory activity greater than that of the 1,3,4thiadiazole derivatives: $(\mathbf{6 a}>11 \mathrm{a}, \mathbf{6 b}>\mathbf{1 1 b}$ and $\mathbf{6 i}>\mathbf{1 1 f})$.

\section{For the 1,3-thiazole ring $6 a-i$}

The in vitro inhibitory activity of 4-methyl-thiazole derivative 6a was greater than that of 4-(2-thienyl)thiazole derivative $\mathbf{6 i}$.

The introduction of an electron-donating group (methyl or methoxy groups) enhanced the antitumor activity. In contrast, the introduction of the electron-withdrawing group (chlorine or bromine or nitro group) at $\mathrm{C} 4$ of the phenyl group at position 4 in the 1,3-thiazole ring decreased the activity: (6d, $6 \mathbf{b}>\mathbf{6 f}, \mathbf{6 e}, \mathbf{6 g})$.

The in vitro inhibitory activity of the 4-tolyl-thiazole derivative 6b was greater than that of 3-tolyl-thiazole derivative $\mathbf{6 c}$.

The in vitro inhibitory activity of the 4-chlorophenyl-thiazole derivative $6 \mathbf{e}$ was greater than that of 2,4-dichlorophenyl-thiazole derivative $\mathbf{6 h}$.

\section{For 1,3,4-thiadiazoles 11a-f}

The in vitro inhibitory activity of 5-substituted thiadiazoles was in the order of $\mathrm{CONHPh}>\mathrm{COOEt}>\mathrm{CH}_{3} \mathrm{CO}>2$-thienyl: $(11 \mathrm{e}>11 \mathrm{c}>$ 11a $>$ 11f).

The in vitro inhibitory activity of 4-methyl-thiadiazole derivative 11a was greater than that of 4-(2-thienyl)thiadiazole derivative $\mathbf{1 1 f}$.

Generally, on fixing the substituents at position 5, the electrondonating group (methyl) at $\mathrm{C} 4$ of the phenyl ring enhances the antitumor activity, while the electron-withdrawing group (chlorine) decreases the activity: $(\mathbf{1 1 b}>\mathbf{1 1 a}$ and $11 \mathbf{d}>\mathbf{1 1 c})$.

\section{CONCLUSION}

A new, efficient, and regioselective method for the preparation of novel 3-azolyl-coumarins by reaction of 3-acetyl-6-methyl$2 \mathrm{H}$-chromen-2-one, thiosemicarbazide, or methyl hydrazinecarbodithioate and the appropriate hydrazonoyl halides under ultrasound irradiation at ambient temperature in a short time and high yields was developed and discussed. The assigned structure for all the newly synthesized compounds was elucidated by elemental and spectral analysis data. Moreover, the new compounds were tested in vitro against the HEPG2-1 cell line using the MTT viability assay. Compounds $\mathbf{6 b}, \mathbf{6 d}$, and $\mathbf{1 1} \mathbf{e}$ have promising activities 
$\left(\mathrm{IC}_{50}\right.$ value of $0.43 \pm 0.66,0.29 \pm 0.45$, and $0.49 \pm 0.38 \mu \mathrm{M}$, respectively), compared with doxorubicin standard drug $\left(\mathrm{IC}_{50}\right.$ value of $0.31 \pm 0.48 \mu \mathrm{M})$.

\section{DATA AVAILABILITY STATEMENT}

The original contributions presented in the study are included in the article/Supplementary Material. Further inquiries can be directed to the corresponding author.

\section{AUTHOR CONTRIBUTIONS}

Formal analysis: SG, HA, and LA-A. Funding acquisition: LA-A and LA. Methodology: SG and HA. Project administration: SG and LA. Resources: SG and HA. Writing-original draft: SG, LA,

\section{REFERENCES}

Abbas, I. M., Gomha, S. M., Elneairy, M. A. A., Elaasser, M. M., and Mabrouk, B. K. H. (2015). Antimicrobial and Anticancer Evaluation of a Novel Synthetic Tetracyclic System Obtained by Dimroth Rearrangement. J. Serb. Chem. Soc. 80, 1251-1264. doi:10.2298/JSC141222022G

Alshabanah, L. A., Gomha, S. M., Al-Mutabagani, L. A., Abolibda, T. Z., El-Ghany, N. A. A., El-Enany, W. A. M. A., et al. (2021). Cross-Linked Chitosan/MultiWalled Carbon Nanotubes Composite as Ecofriendly Biocatalyst for Synthesis of Some Novel Benzil Bis-Thiazoles. Polymers 13, 1728. doi:10.3390/ polym 13111728

Bachman, M., Mann, S. E., and Sheppard, T. D. (2012). Rapid Synthesis of Highly Functionalised $\alpha$-amino Amides and Medium Ring Lactones Using Multicomponent Reactions of Amino Alcohols and Isocyanides. Org. Biomol. Chem. 10, 162-170. doi:10.1039/c1ob06534c

Bhattarai, N., Kumbhar, A. A., Pokharel, Y. R., and Yadav, P. N. (2021). Anticancer Potential of Coumarin and its Derivatives. Mrmc 21, 2996-3029. doi:10.2174/ 1389557521666210405160323

Bhole, R. P., and Bhusari, K. P. (2010). Synthesis and Antitumor Activity of (4Hydroxyphenyl)[5-Substituted Alkyl/aryl)-2-Thioxo-1,3,4-Thiadiazol-3-Yl] methanone and [(3,4-Disubstituted)-1,3-Thiazol-2ylidene $]-4$ Hydroxybenzohydrazide. Med. Chem. Res. 20, 695-704. doi:10.1007/s00044010-9371-9

Cravotto, G., and Cintas, P. (2006). Power Ultrasound in Organic Synthesis: Moving Cavitational Chemistry from Academia to Innovative and Large-Scale Applications. Chem. Soc. Rev. 35, 180-196. doi:10.1039/b503848k

Cravotto, G., Fokin, V. V., Garella, D., Binello, A., Boffa, L., and Barge, A. (2010). Ultrasound-Promoted Copper-Catalyzed Azide-Alkyne Cycloaddition. J. Comb. Chem. 12, 13-15. doi:10.1021/cc900150d

Dawood, K. M., and Gomha, S. M. (2015). Synthesis and Anti-cancer Activity of 1,3,4-Thiadiazole and 1,3-Thiazole Derivatives Having 1,3,4-Oxadiazole Moiety. J. Heterocyclic Chem. 52, 1400-1405. doi:10.1002/jhet.2250

Ekowati, H., Astuti, I., and Mustofa, M. (2010). Anticancer Activity of Calanone on Hela Cell Line. Indo. J. Chem. 10, 240-244. doi:10.22146/ijc.21467

Emami, S., and Dadashpour, S. (2015). Current Developments of Coumarin-Based Anti-cancer Agents in Medicinal Chemistry. Eur. J. Med. Chem. 102, 611-630. doi:10.1016/j.ejmech.2015.08.033

Fu, C.-C., Hung, T.-C., Su, C.-H., Suryani, D., Wu, W.-T., Dai, W.-C., et al. (2011). Immobilization of Calcium Oxide onto Chitosan Beads as a Heterogeneous Catalyst for Biodiesel Production. Polym. Int. 60, 957-962. doi:10.1002/pi.3031

Geisler, J., Sasano, H., Chen, S., and Purohit, A. (2011). Steroid Sulfatase Inhibitors: Promising New Tools for Breast Cancer Therapy? Steroid Biochem. Mol. Biol. 125, 39-45. doi:10.1016/j.jsbmb.2011.02.002

Gomes, J. P. M., Cardoso, C. R. P., Varanda, E. A., Molina, J.-M., Fernandez, M. F., Olea, N., et al. (2011). Antitumoral, Mutagenic and (Anti)estrogenic Activities and HA. Writing-review and editing: HA and SG. All the authors approved the final version.

\section{FUNDING}

This research project was funded by the Deanship of Scientific Research, Princess Nourah bint Abdulrahman University, through the Program of Research Project Funding after Publication, grant No (PRFA-P-42-7).

\section{SUPPLEMENTARY MATERIAL}

The Supplementary Material for this article can be found online at: https://www.frontiersin.org/articles/10.3389/fchem.2021.762248/ full\#supplementary-material

of Tingenone and Pristimerin. Rev. Bras. Farmacogn. 21, 963-971. doi:10.1590/ s0102-695x2011005000153

Gomha, S., Kheder, N., Abdelhamid, A., and Mabkhot, Y. (2016). One Pot Single Step Synthesis and Biological Evaluation of Some Novel Bis(1,3,4-Thiadiazole) Derivatives as Potential Cytotoxic Agents. Molecules 21, 1532. doi:10.3390/ molecules21111532

Gomha, S. M., and Abdel-Aziz, H. M. (2015). Synthesis and Antitumor Activity of 1,3,4-Thiadiazole Derivatives Bearing Coumarine Ring. Heterocycles 91, 583-592. doi:10.3987/com-14-13146

Gomha, S. M., Abdelaziz, M. R., Abdel-aziz, H. M., and Hassan, S. A. (2017). Green Synthesis and Molecular Docking of Thiazolyl-Thiazole Derivatives as Potential Cytotoxic Agents. Mrmc 17, 805-815. doi:10.2174/ 1389557516666161223154539

Gomha, S. M., Abdelhady, H. A., Hassain, D. Z., Abdelmonsef, A. H., El-Naggar, M., Elaasser, M. M., et al. (2021). Thiazole-Based Thiosemicarbazones: Synthesis, Cytotoxicity Evaluation and Molecular Docking Study. Dddt Vol. 15, 659-677. doi:10.2147/dddt.s291579

Gomha, S. M., Abdelrazek, F. M., Abdelrahman, A. H., Metz, P., Mohsen, A., and Sayed, M. A. (2017). A Facile Synthesis and Drug Design of Some New Heterocyclic Compounds Incorporating Pyridine Moiety and Their Antimicrobial Evaluation. Lett. Drug Des. Disc. 14, 752-762. doi:10.2174/ 1570180814666161128120240

Gomha, S. M., Badrey, M. G., and Edrees, M. M. (2016). Heterocyclisation of 2,5-Diacetyl-3,4-Disubstituted-Thieno[2,3-b]Thiophene BisThiosemicarbazones Leading to Bis-Thiazoles and Bis-1,3,4Thiadiazoles as Anti-breast Cancer Agents. J. Chem. Res. 40, 120-125. doi:10.3184/174751916x14537182696214

Gomha, S. M., Riyadh, S. M., Mahmmoud, E. A., and Elaasser, M. M. (2015). Synthesis and Anticancer Activities of Thiazoles, 1,3-Thiazines, and Thiazolidine Using Chitosan-Grafted-Poly(vinylpyridine) as Basic Catalyst. Heterocycles 91, 1227. doi:10.3987/COM-15-13210

Gomha, S. M., Riyadh, S. M., Mahmmoud, E. A., and Elaasser, M. M. (2015). Synthesis and Anticancer Activity of Arylazothiazoles and 1,3,4-thiadiazoles Using Chitosan-GraftedPoly(4-Vinylpyridine) as a Novel Copolymer Basic Catalyst. Chem. Heterocycl. Comp. 51, 1030-1038. doi:10.1007/s10593-016-1815-9

Gomha, S. M., and Riyadh, S. M. (2014). Multicomponent Synthesis of Novel Penta-Heterocyclic Ring Systems Incorporating a Benzopyranopyridine Scaffold. Synthsis 46, 258-262. doi:10.1055/s-0033-1340085

Gomha, S. M., Salah, T. A., and Abdelhamid, A. O. (2015). Synthesis, Characterization, and Pharmacological Evaluation of Some Novel Thiadiazoles and Thiazoles Incorporating Pyrazole Moiety as Anticancer Agents. Monatsh. Chem. 146, 149-158. doi:10.1007/s00706-014-1303-9

Gomha, S., Zaki, Y., and Abdelhamid, A. (2015). Utility of 3-Acetyl-6-Bromo-2hChromen-2-One for the Synthesis of New Heterocycles as Potential Antiproliferative Agents. Molecules 20, 21826-21839. doi:10.3390/ molecules201219803 
Guibal, E. (2005). Heterogeneous Catalysis on Chitosan-Based Materials: a Review. Prog. Polym. Sci. 30, 71-109. doi:10.1016/ j.progpolymsci.2004.12.001

Gupta, K. C., and Ravi Kumar, M. N. V. (2000). Preparation, Characterization and Release Profiles of pH-Sensitive Chitosan Beads. Polym. Int. 49, 141-146. doi:10.1002/(sici) 1097-0126(200002)49:2<141:aid-pi303>3.0.co;2-0

Jarag, K. J., Pinjari, D. V., Pandit, A. B., and Shankarling, G. S. (2011). Synthesis of Chalcone (3-(4-Fluorophenyl)-1-(4-Methoxyphenyl)prop-2-En-1-One): Advantage of Sonochemical Method over Conventional Method. Ultrason. Sonochem. 18, 617-623. doi:10.1016/j.ultsonch.2010.09.010

Kashyap, A., Adhikari, N., Das, A., Shakya, A., Ghosh, S. K., Singh, U. P., et al. (2018). Review on Synthetic Chemistry and Antibacterial Importance of Thiazole Derivatives. Curr. Drug Discov. Technol. 15, 214-228. doi:10.2174/ 1570163814666170911144036

Kumar, D., Kumar, N. M., Chang, K.-H., and Shah, K. (2010). Synthesis and Anticancer Activity of 5-(3-Indolyl)-1,3,4-Thiadiazoles. Eur. J. Med. Chem. 45, 4664-4668. doi:10.1016/j.ejmech.2010.07.023

Li, J., Wang, X.-L., Fang, Y.-C., and Wang, C.-Y. (2010). Tephrosin-induced Autophagic Cell Death in A549 Non-small Cell Lung Cancer Cells. J. Asian Nat. Prod. Res. 12, 992-1000. doi:10.1080/10286020.2010.513034

Liaras, K., Fesatidou, M., and Geronikaki, A. (2018). Thiazoles and Thiazolidinones as COX/LOX Inhibitors. Molecules 685, 23. doi:10.3390/molecules23030685

Lin, M.-H., Cheng, C.-H., Chen, K.-C., Lee, W.-T., Wang, Y.-F., Xiao, C.-Q., et al. (2014). Induction of ROS-independent JNK-Activation-Mediated Apoptosis by a Novel Coumarin-Derivative, DMAC, in Human colon Cancer Cells. Chem. Bio. Int. 218, 42-49. doi:10.1016/j.cbi.2014.04.015

Luzina, E. L., and Popov, A. V. (2009). Synthesis and Anticancer Activity of $\mathrm{N}$-Bis(trifluoromethyl)alkyl-N'-Thiazolyl and N-Bis(trifluoromethyl)alkyl-N'Benzothiazolyl Ureas. Eur. J. Med. Chem. 44, 4944-4953. doi:10.1016/ j.ejmech.2009.08.007

Mamaghani, M., and Hossein Nia, R. (2021). A Review on the Recent Multicomponent Synthesis of Pyranopyrazoles. Polycyclic Aromatic Compd. 41, 223-291. doi:10.1080/10406638.2019.1584576

Marcaccini, S., Miguel, D., Torroba, T., and García-Valverde, M. (2003). 1,4Thiazepines, 1,4-Benzothiazepin-5-Ones, and 1,4-Benzothioxepin Orthoamides via Multicomponent Reactions of Isocyanides. J. Org. Chem. 68, 3315-3318. doi:10.1021/jo026614z

Matysiak, J., and Opolski, A. (2006). Synthesis and Antiproliferative Activity of N-Substituted 2-Amino-5-(2,4-Dihydroxyphenyl)-1,3,4-Thiadiazoles. Bioorg. Med. Chem. 14, 4483-4489. doi:10.1016/j.bmc.2006.02.027

Mavrova, A. T., Wesselinova, D., Tsenov, Y. A., and Denkova, P. (2009). Synthesis, Cytotoxicity and Effects of Some 1,2,4-triazole and 1,3,4-thiadiazole Derivatives on Immunocompetent Cells. Eur. J. Med. Chem. 44, 63-69. doi:10.1016/ j.ejmech.2008.03.006

Nishino, H., Okuyama, T., Takata, M., Shibata, S., Tokuda, H., Takayasu, J., et al. (1990). Studies on the Anti-tumor-promoting Activity of Naturally Occurring Substances. IV. Pd-II $[(+)$ anomalin, (+)praeruptorin B], a Seselin-type Coumarin, Inhibits the Promotion of Skin Tumor Formation by 12-OTetradecanoylphorbol-13-Acetate in 7,12-Dimethylbenz[a]anthraceneInitiated Mice. Carcinogenesis 11, 1557-1561. doi:10.1093/carcin/11.9.1557

Pizzuti, L., Martins, P. L. G., Ribeiro, B. A., Quina, F. H., Pinto, E., Flores, A. F. C., et al. (2010). Efficient Sonochemical Synthesis of Novel 3,5-Diaryl-4,5Dihydro-1h-Pyrazole-1-Carboximidamides. Ultrason. Sonochem. 17, 34-37. doi:10.1016/j.ultsonch.2009.06.013

Popsavin, M., Spaić, S., Svirčeva, M., Kojić, V., Bogdanović, G., and Popsavin, V. (2007). Synthesis and Antitumour Activity of New Tiazofurin Analogues Bearing a 2,3-anhydro Functionality in the Furanose Ring. Bioorg. Med. Chem. Lett. 17, 4123-4127. doi:10.1016/j.bmcl.2007.05.050

Qin, Y., Zhao, W., Yang, L., Zhang, X., and Cui, Y. (2012). Chitosan-Based Heterogeneous Catalysts for Enantioselective Michael Reaction. Chirality 24, 640-645. doi:10.1002/chir.22058
Saidu, N. E. B., Valente, S., Bana, E., Kirsch, G., Bagrel, D., and Montenarh, M. (2012). Coumarin Polysulfides Inhibit Cell Growth and Induce Apoptosis in HCT116 colon Cancer Cells. Bioorg. Med. Chem. 20, 1584-1593. doi:10.1016/ j.bmc.2011.12.032

Shaabani, A., Soleimani, E., and Khavasi, H. R. (2008). Synthesis of 1Aminoimidazo[5,1-A] isoquinolinium Salts Based on Multicomponent Reactions of Isocyanides. J. Comb. Chem. 10, 442-446. doi:10.1021/ cc700196h

Shawali, A. S., and Gomha, S. M. (2000). A New Entry for Short and Regioselective Synthesis of $[1,2,4]$ Triazolo[4,3-B][1,2,4]-Triazin-7(1h)-Ones. J. Prakt. Chem. 342, 599-604. doi:10.1002/1521-3897(200006)342:6<599:aid-prac599>3.0.co; $2-7$

Singh, B. S., Lobo, H. R., Pinjari, D. V., Jarag, K. J., Pandit, A. B., and Shankarling, G. S. (2013). Comparative Material Study and Synthesis of 4-(4-Nitrophenyl) oxazol-2-Amine via Sonochemical and thermal Method. Ultrason. Sonochem. 20, 633-639. doi:10.1016/j.ultsonch.2012.09.002

Tao, Z., Gomha, S. M., Badrey, M. G., El-Idreesy, T. T., and Eldebss, T. M. A. (2018). Novel 4-Heteroaryl-Antipyrines: Synthesis, Molecular Docking, and Evaluation as Potential Anti-breast Cancer Agents. J. Heterocyclic Chem. 55, 2408-2416. doi:10.1002/jhet.3305

Thakur, A., Singla, R., and Jaitak, V. (2015). Coumarins as Anticancer Agents: A Review on Synthetic Strategies, Mechanism of Action and SAR Studies. Eur. J. Med. Chem. 101, 476-495. doi:10.1016/j.ejmech.2015.07.010

Toan, V. N., Thanh, N. D., Tri, N. M., and Huong, N. T. T. (2020). Synthesis and Biological Screening of Thiosemicarbazones of Substituted 3-acetylcoumarins Having D-Glucose Moiety. Bioorg. Med. Chem. Lett. 30, 127664. doi:10.1016/ j.bmcl.2020.127664

Vosooghi, M., Rajabalian, S., Sorkhi, M., Badinloo, M., Nakhjiri, M., Negahbani, A. S., et al. (2010). Synthesis and Cytotoxic Activity of Some 2-Amino-4-Aryl-3-Cyano-7-(dimethylamino)-4h-Chromenes. Res. Pharm. Sci. 5, 9-14.

Wang, S., Zhao, Y., Zhang, G., Lv, Y., Zhang, N., and Gong, P. (2011). Design, Synthesis and Biological Evaluation of Novel 4-thiazolidinones Containing Indolin-2-One Moiety as Potential Antitumor Agent. Eur. J. Med. Chem. 46, 3509-3518. doi:10.1016/ j.ejmech.2011.05.017

Watile, R. A., and Bhanage, B. M. (2012). Chitosan Biohydrogel Beads: A Recyclable, Biodegradable, Heterogeneous Catalyst for the Regioselective Synthesis of 5-Aryl-2-Oxazolidinones from Carbon Dioxide and Aziridines at Mild Conditions. Ind. J. Chem. 51A, 1354-1360.

Xu, H., Liao, W.-M., and Li, H.-F. (2007). A Mild and Efficient UltrasoundAssisted Synthesis of Diaryl Ethers without Any Catalyst. Ultrason. Sonochem. 14, 779-782. doi:10.1016/j.ultsonch.2007.01.002

Conflict of Interest: The authors declare that the research was conducted in the absence of any commercial or financial relationships that could be construed as a potential conflict of interest.

Publisher's Note: All claims expressed in this article are solely those of the authors and do not necessarily represent those of their affiliated organizations, or those of the publisher, the editors, and the reviewers. Any product that may be evaluated in this article, or claim that may be made by its manufacturer, is not guaranteed or endorsed by the publisher.

Copyright $\odot 2022$ Alshabanah, Al-Mutabagani, Gomha and Ahmed. This is an open-access article distributed under the terms of the Creative Commons Attribution License (CC BY). The use, distribution or reproduction in other forums is permitted, provided the original author(s) and the copyright owner(s) are credited and that the original publication in this journal is cited, in accordance with accepted academic practice. No use, distribution or reproduction is permitted which does not comply with these terms. 